\title{
INSCRIBED SEQUENCES OF SURFACES ASSOCIATED WITH GENERALIZED SEQUENCES OF LAPLACE*
}

\author{
BY \\ G. D. GORE \\ 1. INTRODUCTION
}

The theory of conjugate nets of curves on surfaces in a projective space of $n$ dimensions was generalized by Bompiani $\dagger$ in his theory of systems of curves in conjugacy of type $\nu$. Bompiani also generalized the theory of families of asymptotic curves on surfaces by a theory of families of curves in autoconjugacy of type $\nu$.

A set of transformations for surfaces bearing systems of curves in conjugacy of type $\nu$ was offered by B. Segre $\ddagger$, who also gave a system of transformations for surfaces bearing families of curves in autoconjugacy of type $\nu(\nu>1)$. These transformations produce sequences of surfaces quite analogous to the classical sequences of Laplace. In fact the sequences of surfaces bearing systems of curves in conjugacy of type $\nu$ are generalizations of the classical sequences of Laplace. It is these generalized sequences to which we refer in the title.

It is the purpose of this paper to point out a large class of sequences associated with any given sequence of surfaces, and to examine the transformations which generate certain special sequences in that class. Several of the special sequences which we study in that class are associated with the above mentioned sequences of Segre.

In $\$ 2$, we state the geometric basis for a class of sequences called associated sequences, and define generalized inscribed sequences. These generalized inscribed sequences form a subclass of the above associated sequences. A generalized inscribed sequence is generated by the same kind of transformation as generates the sequence in which it is inscribed. The existence of a large class of the general inscribed sequences is established in $\$ 3$, and a web of inscribed sequences is defined. The existence theorem of $\$ 3$ is applied in $\$ 4$ where a study is made of two classes of sequences of surfaces which are inscribed in

* Presented to the Society, April 7, 1934; received by the editors December 15, 1933.

† E. Bompiani, Sistemi coniugati sulle superficie degli iperspazi, Rendiconti del Circolo Matematico di Palermo, vol. 46 (1922), p. 91.

$\ddagger$ B. Segre, Les systèmes conjugués et autoconjugués d'espèce $\nu$ et leur transformation de Laplace, Annales Scientifiques de l'Ecole Normale Supérieure, (3), vol. 44 (1927), pp. 153-212. 
a given hyperbolic sequence of Segre. Two additional types of sequences, inscribed in a given parabolic sequence of Segre, are considered in $\$ 5$.

As a result of this investigation, the transformations of Laplace and Segre are made available for a much less restricted class of surfaces than the class to which they have been applied heretofore.

In the analytical considerations which follow, a point of a surface in a projective space of $n$ dimensions is represented by $n+1$ coordinates $x_{i}$, denoted by the single symbol $x$. The $x_{i}$ are functions of the curvilinear coordinates $u$ and $v$, and they have as many partial derivatives with respect to $u$ and $v$ as are needed. Partial derivatives are denoted in accordance with the formula

$$
\frac{\partial^{i+i} x}{\partial u^{i} \partial v^{i}}=x^{i j}
$$

\section{INSCRIBED SEQUENCES IN GENERAL}

Consider any sequence $T$ of surfaces

$$
\Sigma, \Sigma_{1}, \Sigma_{2}, \cdots, \Sigma_{i}, \cdots,
$$

which is generated by repeated application of a definite transformation such that any surface $\Sigma_{i+1}$ is a transform of the surface $\Sigma_{i}$ by means of a oneto-one point correspondence. The surfaces of $T$ have in common all of the properties of the initial surface $\Sigma$ which are invariant under the generating transformation. In this sense, they will be called mathematically equivalent surfaces. Let $\omega_{r}$ be any osculant of the surface $\Sigma_{r}$ at a general point $P_{r}$, or let $\omega_{r}$ be any osculant at $P_{r}$ of a curve belonging to a family which lies on the surface $\Sigma_{r}$. In either case, let $\omega_{r+1}$ be the corresponding osculant pertaining to the point $P_{r+1}$ of the surface $\Sigma_{r+1}$. Associated with the $\infty^{2}$ points of $\Sigma_{r}$, there are $\infty^{2}$ osculants $\omega_{r}$, and we shall denote the doubly infinite set by $\Omega_{r}$. Obviously, there is associated with the sequence of surfaces $\Sigma_{i}$ a sequence of sets of osculants $\Omega_{i}$.

Consider a surface $\Sigma_{r}^{\prime}$, whose points are in a one-to-one correspondence with the $\infty^{2}$ osculants $\omega_{r}$, pertaining to the surface $\Sigma_{r}$, in such a manner that each point of the surface $\Sigma_{r}^{\prime}$ is in united position with its corresponding osculant $\omega_{r}$. Let $\Sigma_{r+1}^{\prime}$ be a similarly described surface whose points are in a one-to-one united correspondence with the osculants $\omega_{r+1}$ of the set $\Omega_{r+1}$. The surface $\Sigma_{r+1}^{\prime}$ is a transform of the surface $\Sigma_{r}^{\prime}$, by means of the indirect correspondence which connects them. The surfaces $\cdots, \Sigma_{r}^{\prime}, \Sigma_{r+1}^{\prime}, \cdots$ form a sequence $T^{\prime}$ of surfaces which will be referred to as an associated sequence of the sequence $T$. 
DEFINITION 2.1. If the points of a surface are in a one-to-one correspondence with a set of $\infty^{2}$ linear spaces of $\nu$ dimensions, in such a manner that each point of the surface is in united position with its corresponding linear space, then and only then will the surface be said to be transversal to the set of linear spaces.

Definition 2.2. Let $T$ denote a sequence of surfaces in which the points of each surface $\Sigma_{i}$ are joined in a one-to-one manner to the corresponding points of the adjacent surface $\Sigma_{i+1}$ by a set $\Omega$ of $\infty^{2}$ linear spaces $\omega$ of $\nu$ dimensions, where the spaces $\omega$ are osculating spaces at points of the surface $\Sigma_{i}$ to the curves of $a$ family, which lies on the surface $\Sigma_{i}$, or the spaces $\omega$ are osculants of the surface $\Sigma_{i}$ itself. Let $T^{\prime}$ denote a sequence in which consecutive surfaces are connected in the same manner as those of the sequence $T$. Let the sequence $T^{\prime}$ be related to the sequence $T$ so that each surface $\Sigma_{i}^{\prime}$ of the sequence $T^{\prime}$ is transversal to the set $\Omega_{i}$ of osculants which connect the points of the surfaces $\Sigma_{i}$ and $\Sigma_{i+1}$ of $T$. Under these conditions the sequence $T^{\prime}$ will be said to be inscribed in the sequence $T$. The sequence $T$ will be said to circumscribe the sequence $T^{\prime}$.

The surface $\Sigma_{i}^{\prime}$ of the above inscribed sequence $T^{\prime}$ may belong to a more general class of surfaces than does the surface $\Sigma_{i}$ of the sequence $T$. For this reason, the point differential equations which represent the definition of the surface $\Sigma_{i}^{\prime}$ will, in general, be of a higher order than the differential equations which represent the definition of the surface $\Sigma_{i}$ of the sequence $T$. However, the surfaces of the two sequences are connected by correspondences of the same kind. The analytical forms of the transformations in the two sequences will be the same. By proving the existence of these generalized inscribed sequences associated with known sequences, we extend the application of known transformations to a more general class of surfaces and to their point differential equations.

It is observed that a classical inscribed sequence of Laplace furnishes a special example under Definition 2.2. The following article will establish the existence of inscribed sequences of great generality.

\section{A WEB OF INSCRIBED SEQUENCES}

Before defining a web of inscribed sequences, we shall establish the following basic

TheOREM 3.1. Let $T$ denote a sequence of surfaces in which the points of each surface $\Sigma_{i+1}$ are joined in a one-to-one manner to the corresponding points of the preceding surface $\Sigma_{i}$, by a set $\Omega_{i}$ of $\infty^{2}$ osculating spaces of $\nu$ dimensions belonging to the carves of a family on the surface $\Sigma_{i}$. Let $\Sigma_{r}^{\prime}$ be any surface which is transversal to the set $\Omega_{r}$ of osculating spaces $\omega_{r}$. Then it follows that the trans-. versal surface $\Sigma_{r}^{\prime}$ belongs to a sequence $T^{\prime}$ of surfaces which is inscribed in the given sequence $T$. 
Consider a surface $\Sigma$, and on it a family $F$ of curves. Let $\lambda$ denote the curve of the family which passes through the generating point $P$ of the surface $\Sigma$. Denote by $\omega$ the osculating space of $\nu$ dimensions at the point $P$ to the curve $\lambda$. Let $\Sigma^{\prime}$ and $\Sigma^{\prime \prime}$ denote two surfaces which are transversal to the set $\Omega$ of $\infty^{2}$ osculating spaces $\omega$ pertaining to the $\infty^{2}$ points of the curves in the family $F$. Let $P^{\prime}$ and $P^{\prime \prime}$ denote the points of intersection of the surfaces $\Sigma^{\prime}$ and $\Sigma^{\prime \prime}$ respectively with the osculating space $\omega$. As the point $P$ moves along the curve $\lambda$ on the surface $\Sigma$, the points $P^{\prime}$ and $P^{\prime \prime}$ generate two curves, $\lambda^{\prime}$ and $\lambda^{\prime \prime}$ respectively, on the surfaces $\Sigma^{\prime}$ and $\Sigma^{\prime \prime}$. Denote by $\omega^{\prime}$ and $\omega^{\prime \prime}$ the osculating spaces of $\nu$ dimensions to the curves $\lambda^{\prime}$ and $\lambda^{\prime \prime}$ at the respective points $P^{\prime}$ and $P^{\prime \prime}$. We shall show that the osculants $\omega^{\prime}$ and $\omega^{\prime \prime}$ intersect in a point.

Let $x(u, v)$ be the coordinates of the generating point of the above surface $\Sigma$. Let the curves of the above family $F$ be chosen as the parametric $u$-curves. Choose any other family of curves as the $v$-curves. The osculating space of $\nu$ dimensions $\omega$ to the $u$-curve at the point $P$ is determined by $\nu+1$ points, whose coordinates are $x$ and the first $\nu$ derivatives of $x$ with respect to $u$. Since the generating point $P^{\prime}$ of the surface $\Sigma^{\prime}$ is in contact with the osculant $\omega$, the coordinates $y$ of the point $P^{\prime}$ can be expressed as

$$
y=\sum_{i=0}^{\infty} \alpha_{i 0} x^{i 0}
$$

For a similar reason, the coordinates $z$ of the generating point $P^{\prime \prime}$ of the surface $\Sigma^{\prime \prime}$ are

$$
z=\sum_{i=0}^{\nu} \beta_{i 0} x^{i 0} .
$$

The osculating spaces of $\nu$ dimensions $\omega^{\prime}$ and $\omega^{\prime \prime}$ to the $u$-curves at $P^{\prime}$ and $P^{\prime \prime}$ are determined by two sets of points, whose coordinates are $y$ and the first $\nu$ derivatives of $y$, and $z$ with the first $\nu$ derivatives of $z$ with respect to $u$. We exhibit these as follows:

$$
\begin{aligned}
y^{i 0}=\sum_{j=0}^{i+\nu} \alpha_{j 0}^{(i 0)} x^{j 0} & (i=0,1,2, \cdots, \nu), \\
z^{i 0}=\sum_{j=0}^{i+\nu} \beta_{j 0}^{(i 0)} x^{j 0} & (i=0,1,2, \cdots, \nu) .
\end{aligned}
$$

The $2 \nu+2$ coordinates $y^{i 0}$ and $z^{i 0}$, on the left of equations (3.3) and (3.4), are expressed linearly in terms of the $2 \nu+1$ functions $x^{j 0}$. Hence there exists a linear relation among the $z^{i 0}$ and the $y^{i 0}$. That relation will be indicated as 


$$
\sum_{j=0}^{\nu} \theta_{j 0} y^{j 0}=\sum_{j=0}^{\nu} \phi_{j 0} z^{j 0}
$$

Equation (3.5) indicates that the osculating space $\omega^{\prime}$ intersects the osculating space $\omega^{\prime \prime}$ in a point $P_{1}^{\prime}$, the coordinates of which are given by either the right or left member of (3.5). These coordinates are denoted as

$$
y_{1}=\sum_{j=0}^{\nu} \phi_{j 0} z^{j 0} .
$$

The surface generated by the point $P_{1}^{\prime}$ will be denoted by $\Sigma_{1}^{\prime}$.

The above facts justify

LEMMA 3.1. Let $\omega$ be the osculating space of $\nu$ dimensions at the point $P$ to a curve $\lambda$ of a family of curves on a surface $\Sigma$. Let $\Sigma^{\prime}$ and $\Sigma^{\prime \prime}$ be two surfaces which are transversal to the set $\Omega$ of $\infty^{2}$ osculants $\omega$, pertaining to the $\infty^{2}$ points of the surface $\Sigma$. Let $\lambda^{\prime}$ and $\lambda^{\prime \prime}$ be the curves on $\Sigma^{\prime}$ and $\Sigma^{\prime \prime}$ respectively which correspond to the curve $\lambda$ on $\Sigma$. Then it follows that the osculating space of $\nu$ dimensions $\omega^{\prime}$ to the curve $\lambda^{\prime}$ at a point $P^{\prime}$ of the surface $\Sigma^{\prime}$, and the osculating space of $\nu$ dimensions $\omega^{\prime \prime}$ to the curve $\lambda^{\prime \prime}$ at the point $P^{\prime \prime}$ of the surface $\Sigma^{\prime \prime}$, intersect in a point $P_{1}^{\prime}$ which generates a surface $\Sigma_{1}^{\prime}$.

From this lemma, Theorem 3.1 can be obtained directly by assuming that the surface $\Sigma^{\prime \prime}$ of the lemma is a transform $\Sigma_{1}$ of the surface $\Sigma$, and that the surfaces $\Sigma$ and $\Sigma_{1}$ belong to a sequence of mathematically equivalent surfaces

$$
\Sigma, \Sigma_{1}, \Sigma_{2}, \cdots, \Sigma_{i}, \cdots .
$$

Since the surface $\Sigma_{1}^{\prime}$ bears the same relation to the assumed surface $\Sigma_{1}$ as the surface $\Sigma^{\prime}$ bears to the surface $\Sigma$, and since the surfaces $\Sigma$ and $\Sigma_{1}$ are mathematically equivalent, it follows that the surfaces $\Sigma^{\prime}$ and $\Sigma_{1}^{\prime}$ are also mathematically equivalent. That is, the surface $\Sigma_{1}^{\prime}$ is a transform of the surface $\Sigma^{\prime}$. Also, since the surface $\Sigma_{1}$ is transformable into the surface $\Sigma_{2}$ of the sequence (3.7), by a repetition of the above argument, it follows that the surface $\Sigma_{1}^{\prime}$ is transformable in the same manner into a surface $\Sigma_{2}^{\prime}$ of the sequence

$$
\Sigma^{\prime}, \Sigma_{1}^{\prime}, \Sigma_{2}^{\prime}, \cdots, \Sigma_{i}^{\prime}, \cdots \text {. }
$$

If in equations (3.2), (3.5) and (3.6) we replace the coordinates $z$ by $x_{1}$ of the point $P_{1}$ of the surface $\Sigma_{1}$, we obtain the following relations:

$$
\begin{aligned}
x_{1} & =\sum_{i=0}^{\nu} \beta_{i 0} x^{i 0}, \\
\sum_{j=0}^{\nu} \theta_{j 0} y^{j 0} & =\sum_{j=0}^{\nu} \phi_{j 0} x^{j 0},
\end{aligned}
$$




$$
\begin{aligned}
& y_{1}=\sum_{j=0}^{\nu} \theta_{j 0} y^{j 0}, \\
& y_{1}=\sum_{j=0}^{\nu} \phi_{j 0} x_{1}{ }^{j 0} .
\end{aligned}
$$

Equation (3.12) shows that the surface $\Sigma_{1}^{\prime}$, of point coordinates $y_{1}$, is transversal to the $\infty^{2}$ osculating spaces of $\nu$ dimensions to the $u$-curves of the surface $\Sigma_{1}$. This fact indicates that the sequence (3.8) is inscribed in the sequence (3.7). Equation (3.11) shows that corresponding points of the two surfaces $\Sigma^{\prime}$ and $\Sigma_{1}^{\prime}$, of the sequence (3.8), are joined in a one-to-one manner by the osculating spaces of $\nu$ dimensions of the $u$-curves on the surface $\Sigma^{\prime}$. These facts complete the proof of the theorem.

Equation (3.11) shows that the transformation which generates the inscribed sequence (3.8) is of the same analytical form as the transformation (3.9) which generates the circumscribed sequence (3.7).

The inscribed sequence $T^{\prime}$ of Theorem 3.1 has all of the properties of $T$ which are required by the hypothesis. As a consequence, the theorem is applicable to the sequence $T^{\prime}$, and repeatedly, showing that there is in general an endless aggregate of sequences of surfaces

$$
T^{\prime}, T^{\prime \prime}, T^{\prime \prime \prime}, \cdots,
$$

successively inscribed in a given sequence $T$.

Definition. An aggregate of successively inscribed sequences of the type (3.13) will be called a web of inscribed sequences. The sequence $T$ will be said to be circumscribed about the web.

In a given web, the properties of the surfaces vary from one sequence to the next, but the transformations have the same form for the entire web.

4. SEquences INSCRIBED IN A HYPERbolic SEquenCE OF SEgRe

A surface $\Sigma$ bearing a system of curves in conjugacy of type $\nu$ may be defined as an integral surface of a hyperbolic differential equation*

$$
\sum_{i=0}^{\nu} \sum_{j=0}^{1} A_{i j} x^{i j}=0 \text {. }
$$

Segre's transformation of the First Kind $\dagger$ for the above surface $\Sigma$ has the form

$$
x_{1}=\sum_{i=0}^{\nu} b_{i 0} x^{i 0}
$$

* B. Segre, loc. cit., p. 161.

$\dagger$ B. Segre, loc. cit., p. 169. 
for which the coefficients $b_{i 0}$ are determined, to within a proportionality factor, in terms of the $A_{i j}$ of equation (4.1). It is evident from equation (4.2) that the surface $\Sigma_{1}$, generated by the point having coordinates $x_{1}$, is transversal to the osculating spaces of $\nu$ dimensions to the $u$-curves on the surface $\Sigma$. Corresponding points of the two surfaces $\Sigma$ and $\Sigma_{1}$ are joined in a oneto-one manner by the osculating spaces of $\nu$ dimensions of the $u$-curves of the surface $\Sigma$. By repeated application of the transformation (4.2), a sequence $T_{+}$of surfaces

$$
\Sigma, \Sigma_{1}, \Sigma_{2}, \cdots
$$

is generated.

Since each surface of the sequence (4.3) is an integral surface of a hyperbolic differential equation of the type (4.1), we shall refer to the sequence as a hyperbolic sequence of Segre. That part of the entire sequence which is generated by the Segre transformation of the First Kind will be called the forward or positive branch.

From the foregoing remarks, we verify that the positive branch $T_{+}$of the above hyperbolic sequence of Segre has all of the properties required by the hypothesis of Theorem 3.1. Consequently, the theorem is applicable to any surface which is transversal to the $\infty^{2}$ osculating spaces which connect corresponding points of any pair of consecutive surfaces in the sequence of Segre. From this fact we have

THEOREM 4.1. The positive or forward branch $T_{+}$of a hyperbolic sequence of Segre is circumscribed about a web of inscribed sequences

$$
T_{+}^{\prime}, T_{+}^{\prime \prime}, T_{+}^{\prime \prime \prime}, \cdots \text {. }
$$

We now consider a class of sequences of surfaces inscribed in the inverse or negative branch $T_{-}$of a hyperbolic sequence of Segre. The transformation which takes the above surface $\Sigma$ into its transform $\Sigma_{-1}$ of the negative or inverse branch of the sequence of Segre* is represented by the equation

$$
x_{-1}=\sum_{i=0}^{\nu-1} \sum_{j=0}^{1} C_{i j} x^{i j}
$$

The $x_{-1}$ are the coordinates of the point $P_{-1}$ which generates the surface $\Sigma_{-1}$. The coefficients $C_{i j}$ are determined to within a proportionality factor, in terms of the coefficients $A_{i j}$ of equation (4.1).

From the terms of equation (4.5) we observe that the point $P_{-1}$ lies in the sum-space of $2 \nu-1$ dimensions, formed by the osculating space of $\nu-1 \mathrm{di}$ mensions to the $u$-curve at the point $P$ of $\Sigma$, and by the osculating space of

* B. Segre, loc. cit., p. 183. 
$\nu-1$ dimensions to the $u$-curve through the point $(u, v+\Delta v)$, adjacent to $P$. We shall denote this sum-space by $\sigma$, and likewise the corresponding sumspaces at the generating points of the surfaces $\Sigma_{-1}, \Sigma_{-2}, \cdots$ by the corresponding symbols $\sigma_{-1}, \sigma_{-2}, \cdots$.

Let $\Sigma^{\prime}$ denote a surface which is distinct from the two surfaces $\Sigma$ and $\Sigma_{-1}$, but which is transversal to the $\infty^{2}$ osculating spaces $\sigma$ of the surface $\Sigma$. The coordinates $y$ of the generating point $P^{\prime}$ of the surface $\Sigma^{\prime}$ can be expressed as

$$
y=\sum_{i=0}^{n-1} \sum_{j=0}^{1} g_{i j} x^{i j}
$$

in which the $g_{i j}$ are arbitrary except that they are distinct from the $C_{i j}$ of equation (4.5).

On examining the two osculating sum-spaces $\sigma_{-1}$ and $\sigma^{\prime}$ of the surfaces $\Sigma_{-1}$ and $\Sigma^{\prime}$, we find, as will be shown analytically in the next paragraph, that they intersect in a point $P_{-1}^{\prime}$. The point $P_{-1}^{\prime}$ generates a surface $\Sigma_{-1}^{\prime}$ which is transversal to the $\infty^{2}$ osculating spaces $\sigma_{-1}$. It is also transversal to the $\infty^{2}$ osculants $\sigma^{\prime}$ of the surface $\Sigma^{\prime}$. From the mathematical equivalence of the surfaces $\Sigma$ and $\Sigma_{-1}$ and by the fact that the surface $\Sigma_{-1}^{\prime}$ bears the same relation to the surface $\Sigma_{-1}$ as the surface $\Sigma^{\prime}$ bears to $\Sigma$, it follows that the two surfaces $\Sigma^{\prime}$ and $\Sigma_{-1}^{\prime}$ are mathematically equivalent. The surface $\Sigma_{-1}^{\prime}$ is a transform of the surface $\Sigma^{\prime}$, and corresponding points of the two surfaces are joined by the $\infty^{2}$ osculants $\sigma^{\prime}$ of the $u$-curves on the surface $\Sigma^{\prime}$. These facts justify

THEOREM 4.2. Let $\Sigma$ and $\Sigma_{-1}$ denote two consecutive surfaces of the inverse or negative branch $T_{-1}$ of a hyperbolic sequence of Segre. Let $\Sigma^{\prime}$ be any surface which is distinct from the surfaces $\Sigma$ and $\Sigma_{-1}$, and which is transversal to the $\infty^{2}$ osculating sum-spaces $\sigma$ joining corresponding points of $\Sigma$ and $\Sigma_{-1}$. It follows that the surface $\Sigma^{\prime}$ belongs to a sequence $T_{-1}^{\prime}$ of surfaces which is inscribed in the branch $T_{-1}$ of the given sequence of Segre.

For the analytical justification of the above theorem, we exhibit the coordinates of the points which determine the osculating sum-spaces $\sigma^{\prime}$ and $\sigma_{-1}$ at the points $P^{\prime}$ and $P_{-1}$ of the surfaces $\Sigma^{\prime}$ and $\Sigma_{-1}$. By computing derivatives of equations (4.5) and (4.6), we obtain the desired coordinates as the left members of

$$
\begin{aligned}
& x_{-1}^{\lambda \mu}=\sum_{i=0}^{\nu-1+\lambda} \sum_{j=0}^{1+\mu} C_{i j}^{(\lambda \mu)} x^{i j} \quad(\lambda=0,1, \cdots, \nu-1 ; \mu=0,1), \\
& y^{\lambda \mu}=\sum_{i=0}^{\nu-1+\lambda} \sum_{j=0}^{1+\mu} G_{i j}^{(\lambda \mu)} x^{i j} .
\end{aligned}
$$


The left members of (4.7) are $4 \nu$ functions expressed linearly in terms of the $6 \nu-3$ functions $x^{i j}(i=0,1, \cdots, 2 \nu-2 ; j=0,1,2)$. By computing higher derivatives of equation (4.1), it is easily shown that there are $2 \nu-2$ linear relations among the above functions $x^{i j}$. These relations are expressed by the equation

$$
\sum_{i=0}^{\nu+\gamma} \sum_{j=0}^{1+\delta} A_{i j}^{(\gamma \delta)} x^{i j}=0 \quad(\gamma=0,1, \cdots, \nu-2 ; \delta=0,1) .
$$

Hence the above mentioned $4 \nu$ functions of the left members of (4.7) are ultimately expressed in terms of $4 \nu-1$ of the $6 \nu-3$ variables $x^{i j}$. The left members of (4.5) therefore satisfy a linear relation of the form

$$
\sum_{i=0}^{\nu-1} \sum_{j=0}^{1} \theta_{i j} y^{i j}=\sum_{i=0}^{\nu-1} \sum_{j=0}^{1} \psi_{i j} x_{-1}^{i j} .
$$

This equation indicates that the osculating sum-space $\sigma^{\prime}$ of the surface $\Sigma^{\prime}$ intersects the osculating sum-space $\sigma_{-1}$ of $\Sigma_{-1}$ in the point $P_{-1}^{\prime}$, the coordinates $y_{-1}$ of which are obtained from the left member of (4.6) as

$$
y_{-1}=\sum_{i=0}^{\nu-1} \sum_{j=0}^{1} \theta_{i j} y^{i j}
$$

Equation (4.9) exhibits the analytical form of the transformation which generates the inscribed sequence $T_{-1}^{\prime}$ indicated by the above theorem. This transformation is essentially of the same form as the transformation (4.5) which generates the negative branch $T_{-1}$ of the hyperbolic sequence of Segre.

\section{Sequences inscribed in a parabolic sequence of Segre}

A surface $\Sigma$ bearing a family of curves in autoconjugacy of type $\nu$ may be defined as an integral surface of a parabolic differential equation of the type

$$
\sum_{i=0}^{p+1} A_{i 0} x^{i 0}+\sum_{i=0}^{\nu-1} A_{i 1} x^{i 1}=0 . *
$$

The transformation of Segret, which takes the above surface $\Sigma$ into the surface $\Sigma_{1}$ of the positive branch $T_{+}$of a sequence, can be represented by the equation

$$
x_{1}=\sum_{i=0}^{\nu-1} b_{i 0} x^{i 0}
$$$$
(\nu>1) \text {. }
$$

* B. Segre, loc. cit., p. 159.

† B. Segre, loc. cit., p. 206. 
The $b_{i 0}$ are determined, to within a factor, in terms of the coefficients of equation (5.1).

A Segre sequence of surfaces bearing families of curves in autoconjugacy of type $\nu$ will be referred to as a parabolic sequence of Segre.

Equation (5.2) shows that in the positive branch of a parabolic sequence of Segre the corresponding points of two adjacent surfaces $\Sigma$ and $\Sigma_{1}$ are joined, in a one-to-one manner, by the $\infty^{2}$ osculating spaces of $\nu-1$ dimensions to $u$-curves of the surface $\Sigma$. On replacing the index $\nu$ by $\nu-1$ in Theorem 3.1 , we have the resulting

THEOREM 5.1. The positive branch $T_{+}$of a parabolic sequence of Segre is circumscribed about a web of inscribed sequences of surfaces.

Attention will now be given to a class of sequences inscribed in the inverse or negative branch $T_{-}$of a given parabolic sequence of Segre.

The transformation which carries a given surface $\Sigma$ of a parabolic sequence of Segre* into its transform $\Sigma_{-1}$ of the negative branch has the analytic form

$$
x_{-1}=\sum_{i=0}^{\nu} C_{i 0} x^{i 0}+\sum_{i=0}^{\nu-2} C_{i 1} x^{i 1}
$$

in which the $C_{i j}$ are uniquely defined, except for a proportionality factor, in terms of the $A_{i j}$ of (5.1).

Equation (5.3) shows that the point $P_{-1}$, which generates the surface $\Sigma_{-1}$, is in the sum-space formed by the osculating space of $\nu$ dimensions to the $u$-curve through the point $P$ of $\Sigma$ and by the osculating space of $\nu-2$ dimensions to the $u$-curve through the point $(u, v+\Delta v)$ of $\Sigma$. We shall denote this osculating sum-space by $\sigma$, and shall denote the corresponding sum-space of the surface $\Sigma_{-1}$ by $\sigma_{-1}$, etc.

Let $\Sigma^{\prime}$ represent any surface, distinct from the surfaces $\Sigma$ and $\Sigma_{-1}$, which is transversal to the osculating sum-spaces $\sigma$ of the surface $\Sigma$. On examining the osculating sum-spaces $\sigma^{\prime}$ and $\sigma_{-1}$ of the surfaces $\Sigma^{\prime}$ and $\Sigma_{-1}$ we find, as will be demonstrated analytically later, that these two sum-spaces intersect in a point $P_{-1}^{\prime}$. The point $P_{-1}^{\prime}$ generates a surface $\Sigma_{-1}^{\prime}$ which is transversal to the osculating sum-spaces $\sigma_{-1}$ and $\sigma^{\prime}$ of the surfaces $\Sigma_{-1}$ and $\Sigma^{\prime}$. The surface $\Sigma_{-1}^{\prime}$ bears the same relation to the surface $\Sigma_{-1}$ as the surface $\Sigma^{\prime}$ bears to the surface $\Sigma$. Since the surfaces $\Sigma$ and $\Sigma_{-1}$ are mathematically equivalent, it follows that the surfaces $\Sigma^{\prime}$ and $\Sigma_{-1}^{\prime}$ are mathematically equivalent. The surface $\Sigma_{-1}^{\prime}$ is a transform of the surface $\Sigma^{\prime}$, and corresponding points of the

* B. Segre, loc. cit., p. 209. 
two surfaces are joined by the osculating sum-spaces $\sigma^{\prime}$ at points of the surface $\Sigma^{\prime}$. From these facts we have

THEOREM 5.2. Let $\Sigma$ and $\Sigma_{-1}$ be any two consecutive surfaces in the inverse or negative branch $T_{-}$of a parabolic sequence of Segre. Let $\Sigma^{\prime}$ be any third surface which is transversal to the $\infty^{2}$ connecting sum-spaces $\sigma$ pertaining to the surface $\Sigma$. Then it follows that the surface $\Sigma^{\prime}$ belongs to a branch $T_{-1}^{\prime}$ of a sequence of surfaces inscribed in the given sequence of Segre.

To justify the above theorem analytically we exhibit the coordinates of the points which determine the osculating sum-space $\sigma_{-1}$ at the point $P_{-1}$ of the surface $\Sigma_{-1}$. By taking derivatives of the $x_{-1}$, as expressed in (5.3), we obtain

$$
\begin{array}{lr}
x_{-1}^{\lambda 0}=\sum_{i=0}^{\nu+\lambda} C_{i 0}^{(\lambda 0)} x^{i 0}+\sum_{i=0}^{\nu-2+\lambda} C_{i 1}^{(\lambda 0)} x^{i 1} & (\lambda=0,1, \cdots, \nu), \\
x_{-1}^{\mu 1}=\sum_{i=0}^{\nu+\mu} \sum_{j=0}^{1} g_{i j}^{(\mu 1)} x^{i j}+\sum_{i=0}^{\nu-2+\lambda} \sum_{j=0}^{2} h_{i j}^{(\mu 1)} x^{i j} & (\mu=0,1, \cdots, \nu-2) .
\end{array}
$$

In a similar manner the coordinates $y$ of the point $P^{\prime}$ and the remaining points which determine the sum-space $\sigma^{\prime}$ at the point $P^{\prime}$ of the surface $\Sigma^{\prime}$ can be displayed in the form

$$
\begin{array}{lr}
y^{\lambda 0}=\sum_{i=0}^{\nu+\lambda} M_{i 0}^{(\lambda 0)} x^{i 0}+\sum_{i=0}^{\nu-2+\lambda} M_{i 1}^{(\lambda 0)} x^{i 1} & (\lambda=0,1, \cdots, \nu), \\
y^{\mu 1}=\sum_{i=0}^{\nu+\mu} \sum_{j=0}^{1} K_{i j}^{(\mu 1)} x^{i i}+\sum_{i=0}^{\nu-2+\lambda} \sum_{j=0}^{2} P_{i j}^{(\mu 1)} x^{i j} & (\mu=0,1, \cdots, \nu-2) .
\end{array}
$$

The left members of (5.4) and (5.5) are $4 \nu$ functions expressed linearly in terms of the $6 \nu-3$ functions $x^{00}, x^{10}, \cdots, x^{2 v, 0} ; x^{01}, x^{11}, \cdots, x^{2 \nu-2,1}$; $x^{02}, \cdots, x^{2 v-4,2}$. But by means of equation (5.1) and its derivatives we have $2 \nu-2$ linear relations among the above $6 \nu-3$ functions. We exhibit the $2 \nu-2$ relations as follows:

$$
\begin{array}{ll}
\sum_{i=0}^{\nu+1+\lambda} A_{i 0}^{(\lambda 0)} x^{i 0}+\sum_{i=0}^{\nu-1+\lambda} A_{i 1}^{(\lambda 0)} x^{i 1}=0 & (\lambda=0,1, \cdots, \nu-1), \\
\sum_{i=0}^{\nu+1+\mu} \sum_{j=0}^{1} B_{i j}^{(\mu 1)} x^{i j}+\sum_{i=0}^{\nu-1+\mu} \sum_{j=1}^{2} D_{i j}^{(\mu 1)} x^{i j}=0 & (\mu=0,1, \cdots, \nu-3) .
\end{array}
$$

By means of the $2 \nu-2$ relations (5.6), the $4 \nu$ left members of equations (5.4) and (5.5) are expressed linearly in terms of $4 \nu-1$ derivatives of $x$. Hence the left members of (5.4) and (5.5) satisfy a linear relation of the form 


$$
\sum_{i=0}^{\nu} \theta_{i 0} y^{i 0}+\sum_{i=0}^{\nu-2} \theta_{i 1} y^{i 1}=\sum_{i=0}^{\nu} \phi_{i 0} x_{-1}^{i 0}+\sum_{i=0}^{v-2} \phi_{i 1} x_{-1}^{i 1} .
$$

This equation shows that the osculating sum-spaces $\sigma^{\prime}$ and $\sigma_{-1}$ meet in a point $P_{-1}$ the coordinates $y_{-1}$ of which are given by the left members, which we exhibit as

$$
y_{-1}=\sum_{i=0}^{p} \theta_{i 0} y^{i 0}+\sum_{i=0}^{n-2} \theta_{i 1} y^{i 1}
$$

The transformation (5.8), which sends the surface $\Sigma^{\prime}$ into the surface $\Sigma_{-1}^{\prime}$, is obviously of the same form as the transformation (5.3) which sends the surface $\Sigma$ into the surface $\Sigma_{-1}$.

Central Y.M.C.A. College,

Chicago, Ill. 